\title{
A Novel Model-Free Intelligent Proportional-Integral Supertwisting Nonlinear Fractional-Order Sliding Mode Control of PMSM Speed Regulation System
}

\author{
Peng Gao $\mathbb{D}^{1,},{ }^{1,2}$ Xiaodong Lv, ${ }^{1}$ Huimin Ouyang, ${ }^{1}$ Lei Mei, ${ }^{1}$ and Guangming Zhang $\mathbb{D}^{1}$ \\ ${ }^{1}$ College of Electrical Engineering and Control Science, Nanjing Tech University, Nanjing 211899, China \\ ${ }^{2}$ College of Electrical Engineering, Tongling University, Tongling 244000, China \\ Correspondence should be addressed to Guangming Zhang; zgm@njtech.edu.cn
}

Received 4 August 2020; Revised 30 August 2020; Accepted 15 September 2020; Published 28 September 2020

Academic Editor: Mohammad Hassan Khooban

Copyright (c) 2020 Peng Gao et al. This is an open access article distributed under the Creative Commons Attribution License, which permits unrestricted use, distribution, and reproduction in any medium, provided the original work is properly cited.

This study proposes a novel model-free intelligent proportional-integral supertwisting nonlinear fractional-order sliding mode control (MF-iPI-ST-NLFOSMC) strategy for permanent magnet synchronous motor (PMSM) speed regulation system. First of all, a model independent intelligent proportional-integral (iPI) control strategy is adopted for the motor speed regulation system. Next, a novel model-free supertwisting nonlinear fractional-order sliding mode control (ST-NLFOSMC) strategy is constructed based on the ultralocal model of PMSM. Meanwhile, a linear extended state observer (LESO) is used to estimate the unknown terms of the ultralocal model. Then, this study presents the novel hybrid MF-iPI-ST-NLFOSMC strategy which integrates the model-free ST-NLFOSMC strategy, the model-free iPI control strategy, and the LESO. Moreover, the stability of the proposed hybrid MF-iPI-ST-NLFOSMC strategy is proved by the Lyapunov stability theorem and fractional-order theory. Finally, the simulations and comparison results verify that the hybrid MF-iPI-ST-NLFOSMC strategy proposed in this paper has better performance than the other model-free controllers in terms of the static characteristic, dynamic characteristic, and robustness.

\section{Introduction}

A permanent magnet synchronous motor (PMSM) has the advantages of simple structure, small size, high efficiency, and high power factor, which has been widely used in many industrial applications [1-4].

By using a PMSM mathematical model, many prior scholars have designed various advanced controllers for the speed system of PMSM efficiently, examples of such algorithms include model predictive control [5], finite-time feedback control [6], adaptive control [7, 8], and $\mathrm{H}$-infinite control [9]. In this paper, we refer to control strategies of the above as the model-related control strategy. However, due to the existence of various uncertainties in real engineering systems, including parameter uncertainties, incomplete modeling dynamics, and unknown external disturbances, it often poses challenges to the above modelrelated control strategies [10-12]. In view of the above challenges and difficulties, many scholars put forward a model-free control strategy based on an ultralocal mode. In recent years, more and more attention has been paid to the model-free control strategy. Fliess and Join [13] proposed an intelligent proportional-integral (iPI) control strategy based on the ultralocal model. The model-free iPI control strategy has been widely used in the control of various systems, such as single-link flexible-joint manipulator [14], DC/DC power buck converter [15], flexible-link manipulator [16], and PMSM [17]. Unfortunately, the main drawback of the iPI control strategy is that the tracking error of the system cannot be guaranteed to zero rapidly. A sliding mode control (SMC) strategy has the advantages of strong robustness and easy implementation, which has been widely studied [18-21]. Reference [22] introduces the sliding mode control (SMC) into the model-free iPI control strategy to overcome this main drawback. A compound control strategy was proposed based on the model-free 
theory, the iPI control, and the SMC, which combines the advantages of the above control strategies in reference [22]. Recently, fractional-order (FO) theory has been widely used in various fields $[23,24]$. At present, the fractionalorder sliding mode control (FOSMC) strategy has become a popular academic research because of its high precision control, strong robustness, and strong flexibility without significantly increasing the difficulty of implementation $[25,26]$. The FOSMC scheme has widely been used in many fields such as robot manipulator [27], doubly fed induction generator-based wind farm [28], PMSM position regulation system [29], microgyroscope [30], stand-alone modern power grids [31], and fractional-order complex systems [32]. However, the conventional FOSMC has the disadvantages of simple and rough signal processing to limit its application [33-35]. We presented the nonlinear fractional-order sliding mode controller (NLFOSMC), which can effectively settle the abovementioned issues [36-38]. The chattering phenomenon will be leaded by the traditional switch control law of the SMC strategy. In order to overcome the inherent chattering problem of the SMC strategy, many methods have been proposed by many scholars. The supertwisting (ST) structure, as one famous high-order SMC, can effectively eliminate the chattering phenomenon [39]. This paper constructs a supertwisting nonlinear fractional-order sliding mode control (STNLFOSMC) strategy based on the NLFOSMC strategy and the ST structure. The authors of references $[40,41]$ proposed linear extended state observer (LESO). The LESO has the advantages of simple structure and needs less information about the controlled object, which has widely been used in many fields, such as multiaxis servo systems [42], intrusion detection in industrial control system [43], networked interconnected motion system [44], and hypersonic vehicles [45].

In order to overcome the above difficulties, inspired by the above studies and our previous researches [36-38], a novel model-free intelligent proportional-integral supertwisting nonlinear fractional-order sliding mode control (MF-iPI-ST-NLFOSMC) strategy is proposed for the PMSM speed regulation system in this study. The proposed control strategy is based on the ultralocal model of the PMSM. The unknown uncertain dynamics of the ultralocal model is estimated by an LESO. The novel hybrid MF-iPI-STNLFOSMC strategy is proposed in this paper which integrates the ST-NLFOSMC strategy, the model-free iPI control strategy, and the LESO. Most importantly, the proposed MFiPI-ST-NLFOSMC strategy not only solves the problem that the error cannot reach zero quickly in the traditional iPI control strategy of the ultralocal mode but also has the advantages of the NLFOSMC and the ST structure.

Compared with previous studies, the main contributions of this article can be summarized as

(1) A hybrid MF-iPI-ST-NLFOSMC strategy is proposed for PMSM speed regulation system, which integrates the ST structure, the NLFOSMC strategy, the iPI control strategy, and the LESO. Especially, the proposed control strategy does not depend on the actual mathematical model of PMSM.

(2) A novel LESO based on the hybrid MF-iPI-STNLFOSMC strategy is proposed for the PMSM drives. The stability of the proposed hybrid MF-iPIST-NLFOSMC strategy is proved by the Lyapunov stability theorem and fractional-order theory.

(3) The simulations and comparison results verify that the proposed hybrid MF-iPI-ST-NLFOSMC strategy has better performance than the other model-free controllers in terms of the static characteristic, dynamic characteristic, and robustness.

The remaining parts of this paper are arranged as follows: In Section 2, some mathematical preliminaries are briefly presented. Control strategies and stability analysis are given in Section 3. The simulation experiment and comparison results are shown in Section 4. Finally, Section 5 concludes the study.

\section{Mathematical Preliminaries}

This section introduces some important knowledge of fractional-order calculus, as well as the traditional mathematical model and the ultralocal model of PMSM.

Definition 1 (see [46, 47]). The Riemann-Liouville fractional derivative and integral of continuous function $f(t)$ are given as follows:

$$
\left\{\begin{array}{l}
D_{t}^{\varepsilon} f(t)=\frac{1}{\Gamma(m-\varepsilon)} \frac{\mathrm{d}^{m}}{\mathrm{~d} t^{m}} \int_{t_{0}}^{t} \frac{f(\tau)}{(t-\tau)^{1-m+\varepsilon}} \mathrm{d} \tau \\
D_{t}^{-u} f(t)=\frac{1}{\Gamma(u)} \int_{t_{0}}^{t} \frac{f(\tau)}{(t-\tau)^{1-u}} \mathrm{~d} \tau \\
\Gamma(z)=\int_{0}^{\infty} e^{-t} t^{z-1} \mathrm{~d} t
\end{array}\right.
$$

where $z \in R ; \varepsilon$ and $u$ are the fractional derivative order and fractional integral order, respectively; and $m-1<\varepsilon<m$; $m \in N$.

The Caputo fractional derivative of order $u$ of a continuous function $f: R^{+} \longrightarrow R$ is defined as follows:

$$
t_{0} D_{t}^{u} f(t)=\left\{\begin{array}{l}
\frac{1}{\Gamma(m-u)} \int_{t_{0}}^{t} \frac{f^{(m)}(\tau)}{(t-\tau)^{u-m+1}} \mathrm{~d} \tau, \quad m-1<u<m, \\
\frac{\mathrm{d}^{m}}{\mathrm{~d} t^{m}} f(t), \quad u=m,
\end{array}\right.
$$

where $m$ is the first integer larger than $u$.

Lemma 1 (see [48]). For the fractional-order integration and differentiation, the following properties hold: 


$$
\begin{aligned}
\frac{d^{m}}{d t^{m}}\left(D_{t}^{\varepsilon} f(t)\right) & =D_{t}^{\varepsilon+m} f(t), \\
D_{t}^{\varepsilon}(a f(t)) & =a D_{t}^{\varepsilon} f(t), \quad a \in R, \\
D_{t}^{\varepsilon}(f(t)+g(t)) & =D_{t}^{\varepsilon} f(t)+D_{t}^{\varepsilon} g(t) .
\end{aligned}
$$

PMSM electromagnetic torque equation is described as follows [49]:

$$
T_{e}=\frac{3}{2} p_{n}\left(\phi_{f} i_{q}(t)+\left(L_{d}-L_{q}\right) i_{d}(t) i_{q}(t)\right),
$$

where $T_{e}$ represents the electromagnetic torque; $\phi_{f}$ is the permanent magnet flux linkage; $p_{n}$ is the pole pairs number; $L_{d}$ and $L_{q}$ represent the inductances of the $d$ and $q$ axes; and $i_{d}(t)$ and $i_{q}(t)$ are the components of armature currents of $d$ and $q$ axes, respectively.

For surface-mount PMSM, $L_{d}=L_{q}=L$ in this paper. The PMSM mathematical model can be gave as [49]

$$
\begin{aligned}
& \left\{\begin{array}{l}
T_{e}-T_{L}=J \dot{\omega}(t)+B \omega(t), \\
T_{e}=\frac{3}{2} p_{n} \phi_{f} i_{q}(t),
\end{array}\right. \\
& \dot{\omega}(t)=\frac{3 p_{n} \phi_{f}}{2 J} i_{q}(t)-\frac{B}{J} \omega(t)-\frac{1}{J} T_{L},
\end{aligned}
$$

where $\omega(t)$ is the actual mechanical speed and $J, T_{L}$, and $B$ are the rotational inertia, the load torque, and the friction coefficient, respectively. follows:

The control objective of this paper can be expressed as

$$
e(t)=\omega_{r}(t)-\omega(t)=0,
$$

where $\omega_{r}(t)$ is the reference speed and $e(t)$ is the speed error.

Definition 2 (see $[13,50]$ ). The PMSM mathematical model can be replaced by the ultralocal model as

$$
\dot{y}(t)=a u(t)+F,
$$

where $y(t)$ and $u(t)$ are the output and input of the ultralocal model, respectively; $a$ is the constant parameter; and $F$ is the unknown term of the system.

The objective of the ultralocal model system being controlled is

$$
e(t)=y_{r}(t)-y(t)=0,
$$

where $y_{r}(t)$ and $e(t)$ are the desired output and the tracking error, respectively.

\section{Control Strategies and Stability Analysis}

3.1. Design of Second-Order LESO. A second-order LESO is chosen to estimate the unknown term of the system and any disturbances in this paper.

According to Definition 2, the mathematical model of the PMSM can be expressed as

$$
\left\{\begin{array}{l}
\dot{x}_{1}=x_{2}+a u \\
\dot{x}_{2}=\ell(t)
\end{array}\right.
$$

where $x_{1}=y(t), x_{2}=F$, and $\ell(t)$ represents the differential of $x_{2}$.

The second-order LESO is designed as [40, 41]

$$
\left\{\begin{array}{l}
e(t)=Z_{21}-y(t) \\
\dot{Z}_{21}=Z_{22}-\beta_{1} e(t)+b_{0} u(t), \\
\dot{Z}_{22}=-\beta_{2} e(t)
\end{array}\right.
$$

where $\beta_{1}$ and $\beta_{2}$ are the positive constants, $b_{0}$ is the design parameter, and $Z_{21}$ and $Z_{22}$ are the estimates of $y(t)$ and $F$, respectively.

\subsection{Design of Control Strategies}

Definition 3 (see $[13,50]$ ). The iPI control strategy is defined as

$$
u_{1}(t)=\frac{1}{a}\left(K_{p} e(t)+K_{i} \int e(t) \mathrm{d} t+\dot{y}_{r}(t)-F\right),
$$

where $K_{p}>0, K_{i}>0$, and $u_{1}(t)$ is the control law of the iPI control strategy.

Substituting (10) into (11), the iPI control strategy can be rewritten as

$$
u_{1}(t)=\frac{1}{a}\left(K_{p} e(t)+K_{i} \int e(t) \mathrm{d} t+\dot{y}_{r}(t)-Z_{22}\right) .
$$

According to Definition 2, (8) and (12), the following equation can be obtained:

$$
\left\{\begin{array}{l}
K_{p} e(t)+K_{i} \int e(t) \mathrm{d} t+\dot{e}(t)+\Delta \mathrm{d}(t)=0 \\
\Delta \mathrm{d}(t)=F-Z_{22} .
\end{array}\right.
$$

Applying the Laplace transformation for (13), the following equation can be gained:

$$
\left(s+K_{p}+\frac{K_{i}}{s}\right) E(s)+\Delta \mathrm{d}(s)-\Delta \mathrm{d}\left(0^{+}\right)=0 .
$$

The steady-state error of the system can be derived from the final value theorem as follows:

$$
e\left(t_{\infty}\right)=\lim _{s \longrightarrow 0} \frac{s^{2}}{s^{2}+K_{p} s+K_{i}}\left(\Delta \mathrm{d}\left(0^{+}\right)-\Delta \mathrm{d}(s)\right) .
$$

As a result of equation (15), the tracking error cannot be quickly equal to zero in the iPI control strategy. A detailed description of the iPI control strategy can be found in references $[13,22,50]$.

According to reference [22], a traditional model-free iPI SMC (MF-iPI-SMC) strategy is designed based on the traditional SMC strategy, the model-free control theory, and the iPI control strategy.

The traditional sliding mode surface $[51,52]$ is given as follows: 


$$
s_{\mathrm{TSM}}(t)=\eta_{1} e(t)+\eta_{2} \int e(t) \mathrm{d} t
$$

where $\eta_{1}$ and $\eta_{2}$ are the positive parameters of the traditional sliding mode surface.

Definition 4. Based on the traditional sliding mode surface, the traditional MF-iPI-SMC strategy is designed in the following format:

$$
\begin{aligned}
& u_{\mathrm{MF}-\mathrm{iPI}-\mathrm{FOSMC}}(t)=u_{1}(t)+u_{2}(t) \\
& =\left\{\begin{array}{l}
\frac{1}{a}\left(K_{p} e(t)+K_{i} \int e(t) \mathrm{d} t+\dot{y}_{r}(t)-Z_{22}\right)+u_{2}(t) \\
u_{2}(t)=u_{21}(t)+u_{22}(t)
\end{array}\right.
\end{aligned}
$$

where

$$
\begin{aligned}
& u_{21}=\frac{1}{a}\left(-K_{p} e(t)-K_{i} \int e(t) \mathrm{d} t\right)+\frac{\eta_{2}}{\eta_{1} a} e(t), \\
& u_{22}=\frac{1}{a}\left(\eta \operatorname{sign}\left(s_{\mathrm{TSM}}(t)\right)\right),
\end{aligned}
$$

where $\eta \in R^{+}$is the positive parameter of the switching control law and $\operatorname{sign}(f(\cdot))=\left\{\begin{array}{ll}1 & f(\cdot)>0, \\ 0 & f(\cdot)=0, \\ -1 & f(\cdot)<0 .\end{array}\right.$.

In what follows, a model-free iPI fractional-order SMC (MF-iPI-FOSMC) strategy is designed based on the FOSMC strategy, the model-free control theory, and the iPI control strategy.

In this paper, the following fractional-order sliding mode surface is chosen as $[53,54]$

$$
s_{\mathrm{FOSM}}(t)=K_{f p} e(t)+K_{f i} D_{t}^{u-1} e(t)+K_{f d} D_{t}^{\varepsilon} e(t),
$$

where $K_{f p}, K_{f i}$, and $K_{f d}$ are the positive parameters of the fractional-order sliding mode surface; $0<\varepsilon, u<1$.

Definition 5. The MF-iPI-FOSMC strategy is designed in the following format:

$$
u_{\mathrm{MF}-\mathrm{iPI}-\mathrm{SMC}}(t)=u_{1}(t)+u_{2}(t)=\left\{\begin{array}{l}
\frac{1}{a}\left(K_{p} e(t)+K_{i} \int e(t) \mathrm{d} t+\dot{y}_{r}(t)-Z_{22}\right)+u_{2}(t), \\
u_{2}(t)=u_{21}(t)+u_{22}(t)
\end{array}\right.
$$

In addition, in order to make the control error of the system rapidly zero, by substituting (20) in (7), the following equation can be obtained:

$$
\dot{e}(t)+a u_{2}+K_{p} e(t)+K_{i} \int e(t) \mathrm{d} t+\Delta \mathrm{d}(t)=0 .
$$

According to Lemma 1, taking derivative of (19), the following can be obtained:

$$
\dot{s}_{\mathrm{FOSM}}(t)=K_{f p} \dot{e}(t)+K_{f i} D_{t}^{u} e(t)+K_{f d} D_{t}^{1+\varepsilon} e(t) .
$$

Substituting (21) in (22), the following equation can be obtained:

$$
\begin{aligned}
& \dot{s}_{\mathrm{FOSM}}(t)=K_{\mathrm{fp}}\left(-a u_{2}-K_{p} e(t)-K_{i} \int e(t) \mathrm{d} t-\Delta d(t)\right) \\
& +K_{\mathrm{fi}} D_{t}^{u} e(t)+K_{\mathrm{fd}} D_{t}^{1+\varepsilon} e(t) .
\end{aligned}
$$

According to (23) and $Z_{22}=F$, the equivalent control law of the MF-iPI-FOSMC strategy can be obtained as

$$
u_{21}=\frac{1}{a}\left(-K_{p} e(t)-K_{i} \int e(t) \mathrm{d} t\right)+\frac{K_{\mathrm{fi}}}{K_{\mathrm{fp}} a} D_{t}^{u} e(t)+\frac{K_{\mathrm{fd}}}{K_{\mathrm{fp}} a} D_{t}^{1+\varepsilon} e(t) .
$$

The switching control law of the MF-iPI-FOSMC strategy is designed as

$$
u_{22}=\frac{1}{a}\left(\eta \operatorname{sign}\left(s_{\mathrm{FOSM}}(t)\right)\right) .
$$

In what follows, a model-free iPI nonlinear fractionalorder SMC (MF-iPI-NLFOSMC) strategy is designed based on the NLFOSMC strategy, the model-free control theory, and the iPI control strategy.

We design the nonlinear fractional-order sliding mode surface as follows:

$$
\begin{aligned}
s_{\mathrm{NLFOSM}}(t)= & K_{n l f p} f a l(e(t))+K_{n l f i} D_{t}^{u-1} f a l(e(t)) \\
& +K_{n l f d} D_{t}^{\varepsilon} f a l(e(t)), \\
\operatorname{fal}(e(t))= & \begin{cases}|e(t)|^{\tilde{\alpha}} \operatorname{sign}(e(t)) & |e(t)|>\widetilde{\delta}, \\
\frac{e(t)}{\widetilde{\delta}^{1-\tilde{\alpha}}} & |e(t)| \leq \widetilde{\delta},\end{cases}
\end{aligned}
$$

where $0<\tilde{\delta} ; 0<\tilde{\alpha}<1$; and $K_{n l f p}, K_{n l f i}$, and $K_{n l f d}$ are the coefficients of the nonlinear fractional-order sliding mode surface. 
Remark 1. The gains of $K_{n l f p}, K_{n l f i}$, and $K_{n l f d}$ should be set to $K_{n l f p} \in R^{+}, K_{n l f i} \in R^{+}$, and $K_{n l f d} \in R^{+} ; 0<\varepsilon, u<1$, and the system stability can be satisfied. The detailed description is given in the Stability Analysis section.

A detailed description of the nonlinear fractional-order sliding mode surface (26) can be found in reference [38].
Figure 1 draws the corresponding curves of the nonlinear function (27) under different parameters [38].

Definition 6. The proposed MF-iPI-NLFOSMC strategy is designed in the following format:

$$
u_{\text {MF-iPI-NLFOSMC }}(t)=u_{1}(t)+u_{2}(t)=\left\{\begin{array}{l}
\frac{1}{a}\left(K_{p} e(t)+K_{i} \int e(t) \mathrm{d} t+\dot{y}_{r}(t)-Z_{22}\right)+u_{2}(t), \\
u_{2}(t)=u_{21}(t)+u_{22}(t) .
\end{array}\right.
$$

According to Lemma 1, taking the derivative of the nonlinear fractional-order sliding mode surface (26), the following can be obtained:

$$
\dot{s}_{\mathrm{NLFOSM}}(t)= \begin{cases}K_{\mathrm{nlfp}} \widetilde{\alpha}|e|^{\tilde{\alpha}-1} \dot{e}(t)+K_{\mathrm{nlfi}} D_{t}^{u} \mathrm{fal}(e(t))+K_{\mathrm{nlfd}} D_{t}^{1+\varepsilon} \mathrm{fal}(e(t)), & \text { if }|e(t)|>\widetilde{\delta}, \\ \left(\frac{K_{\mathrm{nlfp}}}{\widetilde{\delta}^{1-\widetilde{\alpha}}}\right) \dot{e}(t)+K_{\mathrm{nlfi}} D_{t}^{u} \mathrm{fal}(e(t))+K_{\mathrm{nlfd}} D_{t}^{1+\varepsilon} \mathrm{fal}(e(t)), & \text { if }|e(t)| \leq \widetilde{\delta} .\end{cases}
$$

Substituting (21) in (29), the following equation can be obtained as

$$
\dot{s}_{\text {NLFOSM }}(t)= \begin{cases}K_{n l f p} \widetilde{\alpha}|e|^{\widetilde{\alpha}-1}\left(-a u_{2}-K_{p} e(t)-K_{i} \int e(t) \mathrm{d} t-\Delta \mathrm{d}(t)\right)+K_{n l f i} D_{t}^{u} f a l(e(t))+K_{n l f d} D_{t}^{1+\varepsilon} f a l(e(t)), & \text { if }|e(t)|>\widetilde{\delta}, \\ \left(\frac{K_{n l f p}}{\widetilde{\delta}^{1-\widetilde{\alpha}}}\right)\left(-a u_{2}-K_{p} e(t)-K_{i} \int e(t) \mathrm{d} t-\Delta \mathrm{d}(t)\right)+K_{n l f i} D_{t}^{u} \mathrm{fal}(e(t))+K_{n l f d} D_{t}^{1+\varepsilon} f a l(e(t)), & \text { if }|e(t)| \leq \widetilde{\delta} .\end{cases}
$$

According to (30) and $\Delta \mathrm{d}(t)=0$, the equivalent control law of the proposed MF-iPI-NLFOSMC strategy can be obtained as

$$
u_{21}= \begin{cases}\frac{1}{a}\left(-K_{p} e(t)-K_{i} \int e(t) \mathrm{d} t\right)+\frac{K_{\mathrm{nlfi}}}{\widetilde{\alpha}|e|^{\tilde{\alpha}-1} K_{\mathrm{nlfp}} a} D_{t}^{u} \text { fal }(e(t))+\frac{K_{\mathrm{nlfd}}}{\widetilde{\alpha}|e|^{\tilde{\alpha}-1} K_{\mathrm{nlfp}} a} D_{t}^{1+\varepsilon} \mathrm{fal}(e(t)), & \text { if }|(e(t))|>\widetilde{\delta}, \\ \frac{1}{a}\left(-K_{p} e(t)-K_{i} \int e(t) \mathrm{d} t\right)+\frac{K_{\mathrm{nlfi}}}{\left(1 / \widetilde{\delta}^{1-\widetilde{\alpha}}\right) K_{\mathrm{nlfp}} a} D_{t}^{u} \mathrm{fal}(e(t))+\frac{K_{\mathrm{nlfd}}}{\left(1 / \widetilde{\delta}^{1-\widetilde{\alpha}}\right) K_{\mathrm{nlfp}} a} D_{t}^{1+\varepsilon} \text { fal }(e(t)), & \text { if }|(e(t))| \leq \widetilde{\delta} .\end{cases}
$$

The switching control law of the proposed MF-iPINLFOSMC strategy is designed as

$$
u_{22}=\frac{1}{a}\left(\eta \operatorname{sign}\left(s_{\mathrm{NLFOSM}}(t)\right)\right) .
$$




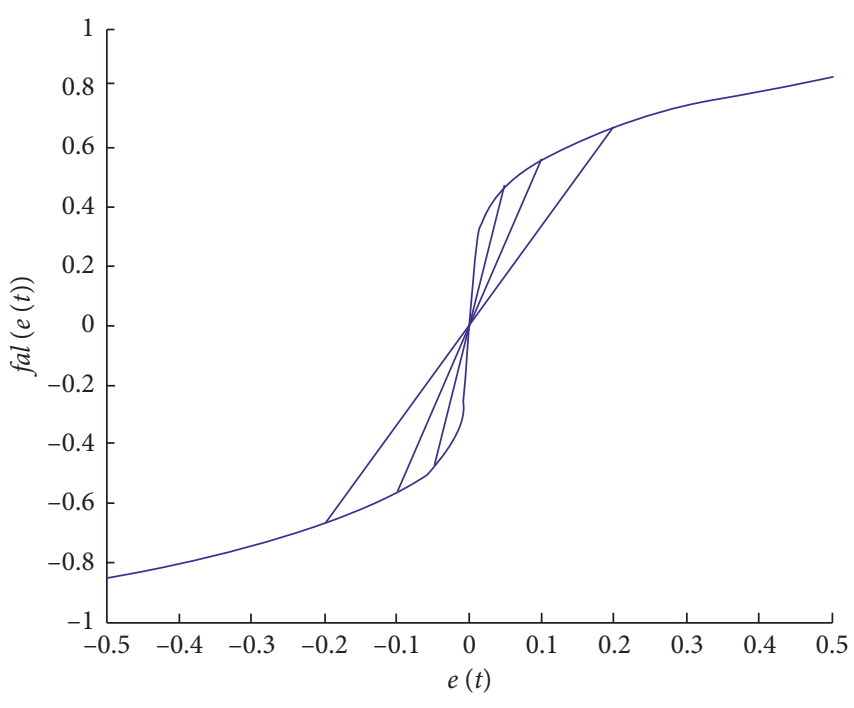

FIGURE 1: Characteristic curves of the nonlinear function fal $(e(t))$.

In what follows, a model-free iPI supertwisting nonlinear fractional-order SMC (MF-iPI-ST-NLFOSMC) strategy is designed based on the ST-NLFOSMC strategy, the modelfree control theory, and the iPI control strategy.

Definition 7. The proposed MF-iPI-ST-NLFOSMC strategy is designed in the following format:

$$
\begin{array}{r}
u_{\mathrm{MF}-\mathrm{iPI}-\mathrm{ST}-\mathrm{NLFOSMC}}(t)=u_{1}(t)+u_{2}(t), \\
=\left\{\begin{array}{l}
\frac{1}{a}\left(K_{p} e(t)+K_{i} \int e(t) \mathrm{d} t+\dot{y}_{r}(t)-Z_{22}\right)+u_{2}(t), \\
u_{2}(t)=u_{21}(t)+u_{22}(t),
\end{array}\right.
\end{array}
$$

where

$$
u_{21}= \begin{cases}\frac{1}{a}\left(-K_{p} e(t)-K_{i} \int e(t) \mathrm{d} t\right)+\frac{K_{\mathrm{nlfi}}}{\widetilde{\alpha}|e|^{\widetilde{\alpha}-1} K_{\mathrm{nlfp}} a} D_{t}^{u} \mathrm{fal}(e(t))+\frac{K_{\mathrm{nlfd}}}{\widetilde{\alpha}|e|^{\widetilde{\alpha}-1} K_{\mathrm{nlfp}} a} D_{t}^{1+\varepsilon} \mathrm{fal}(e(t)), & \text { if }|(e(t))|>\widetilde{\delta}, \\ \frac{1}{a}\left(-K_{p} e(t)-K_{i} \int e(t) \mathrm{d} t\right)+\frac{K_{\mathrm{nlfi}}}{\left(1 / \widetilde{\delta}^{1-\widetilde{\alpha}}\right) K_{\mathrm{nlfp}} a} D_{t}^{u} \mathrm{fal}(e(t))+\frac{K_{\mathrm{nlfd}}}{\left(1 / \widetilde{\delta}^{1-\widetilde{\alpha}}\right) K_{\mathrm{nlfp}} a} D_{t}^{1+\varepsilon} \mathrm{fal}(e(t)), & \text { if }|(e(t))| \leq \widetilde{\delta} .\end{cases}
$$

In order to improve the control effect of the system in the approaching state and eliminate the chattering phenomenon, the following ST structure control law is designed as

$$
u_{22}=\frac{1}{a}\left(k_{1}\left|s_{\mathrm{NLFOSM}}(t)\right|^{(1 / 2)} \operatorname{sign}\left(s_{\mathrm{NLFOSM}}(t)\right)+k_{2} \int \operatorname{sign}\left(s_{\mathrm{NLFOSM}}(t)\right) \mathrm{d} t\right),
$$

where $k_{1}$ and $k_{2}$ are the positive parameters of the ST structure control law.

Remark 2. The gains of $k_{1}$ and $k_{2}$ are set to be larger enough, and the system stability can be satisfied. The detailed description is given in the following section.

A novel field-oriented control of PMSM speed regulation system based on the proposed MF-iPI-ST-NLFOSMC strategy with the second-order LESO is shown in Figure 2. The structural diagram of the proposed MF-iPI-STNLFOSMC strategy is shown in Figure 3.

3.3. Stability Analysis. Motivated by the previous studies [55-57], the stability of the control strategies mentioned in this article is discussed in the following sections.
First, the stability of the LESO will be discussed.

Proof. The LESO (10) can be rewritten as

$$
\left\{\begin{array}{l}
\dot{Z}_{21}=Z_{22}+\beta_{1}\left(y(t)-Z_{21}\right)+b_{0} u(t), \\
\dot{Z}_{22}=\beta_{2}\left(y(t)-Z_{21}\right) .
\end{array}\right.
$$

Let,

$$
\begin{gathered}
A=\left[\begin{array}{cc}
-\beta_{1} & 1 \\
-\beta_{2} & 0
\end{array}\right], \\
B=\left[\begin{array}{ll}
\beta_{1} & b_{0} \\
\beta_{2} & 0
\end{array}\right] .
\end{gathered}
$$

Write (36) in a matrix form as 


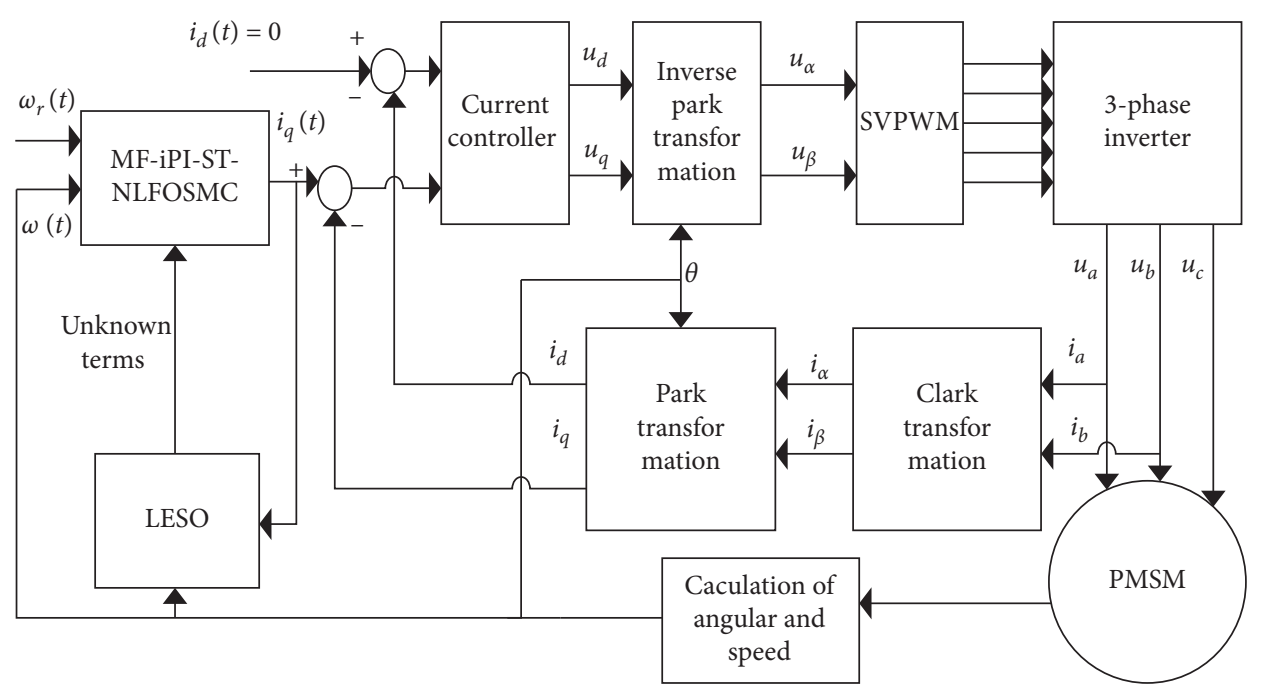

FIgURE 2: The configuration of the novel field-oriented control of PMSM speed regulation system based on the proposed MF-iPI-STNLFOSMC strategy.

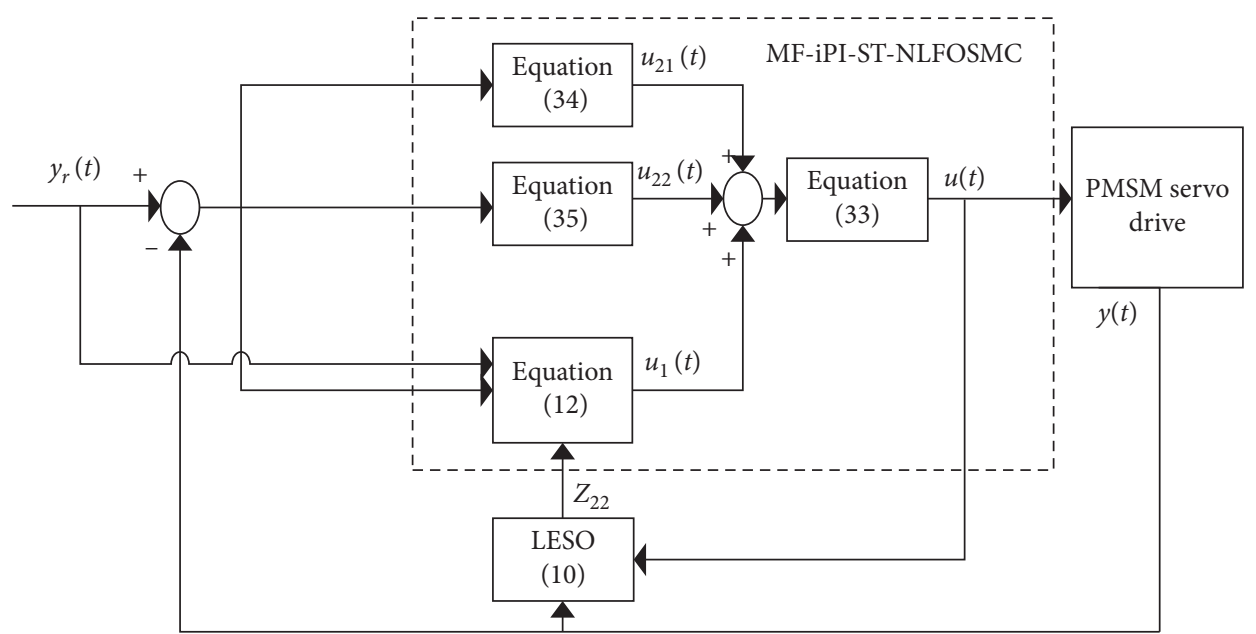

FIGURE 3: Structural diagram of the proposed MF-iPI-ST-NLFOSMC strategy.

$$
\left[\begin{array}{l}
\dot{Z}_{21} \\
\dot{Z}_{22}
\end{array}\right]=A\left[\begin{array}{l}
Z_{21} \\
Z_{22}
\end{array}\right]+B\left[\begin{array}{l}
y(t) \\
u(t)
\end{array}\right]
$$

On the basis of the Lyapunov stability theory, we know that the system (36) is stable.

It means that

$$
\left\{\begin{array}{l}
Z_{21} \longrightarrow y(t), \\
Z_{22} \longrightarrow F .
\end{array}\right.
$$

Therefore, the LESO exhibits stability. A detailed description of the stability of the LESO can be found in reference [57].

In what follows, the stability of the MF-iPI-FOSMC strategy will be discussed.
Proof. Assumption 1. $|\Delta \mathrm{d}(t)|$ value has an upper limit, and the estimation error of the LESO satisfies the following condition:

$$
|\Delta \mathrm{d}(t)|<\psi,
$$

where $\psi$ is a positive constant.

In order to guarantee the stability of the MF-iPI-FOSMC strategy, the Lyapunov function for the MF-iPI-FOSMC strategy is defined as

$$
V=\frac{1}{2} s_{\mathrm{FOSM}}(t)^{2}>0 .
$$

Taking the derivative on both sides of equation (41), the following can be derived: 


$$
\begin{aligned}
\dot{V}= & s_{\mathrm{FOSM}}(t) \cdot \dot{s}_{\mathrm{FOSM}}(t) \\
= & s_{\mathrm{FOSM}}(t)\left(K_{\mathrm{fp}} \dot{e}(t)+K_{\mathrm{fi}} D_{t}^{u} e(t)+K_{\mathrm{fd}} D_{t}^{1+\varepsilon} e(t)\right) \\
= & s_{\mathrm{FOSM}}(t)\left(\begin{array}{c}
\left.K_{f p}\left(-a u_{2}-K_{p} e(t)-K_{i} \int e(t) \mathrm{d} t-\Delta \mathrm{d}(t)\right)\right) \\
+K_{f i} D_{t}^{u} e(t)+K_{\mathrm{fd}} D_{t}^{\varepsilon+1} e(t)
\end{array}\right) \\
= & s_{\mathrm{FOSM}}(t)\left(-K_{\mathrm{fp}} \eta \operatorname{sign}\left(s_{\mathrm{FOSM}}(t)\right)-K_{f p} \Delta \mathrm{d}(t)\right) \\
= & -\operatorname{sign}\left(s_{\mathrm{FOSM}}(t)\right) K_{\mathrm{fp}} \eta s_{\mathrm{FOSM}}(t)-s_{\mathrm{FOSM}}(t) K_{\mathrm{fp}} \Delta \mathrm{d}(t) \\
= & -\eta K_{\mathrm{fp}}\left|s_{\mathrm{FOSM}}(t)\right|-s_{\mathrm{FOSM}}(t) K_{\mathrm{fp}} \Delta \mathrm{d}(t) \\
& \leq-\eta K_{\mathrm{fp}}\left|s_{\mathrm{FOSM}}(t)\right|+\left|s_{\mathrm{FOSM}}(t)\right| K_{\mathrm{fp}}|\Delta \mathrm{d}(t)| .
\end{aligned}
$$

According to Assumption 1 and when $\eta>\psi$ is satisfied, we can get $\dot{V}<0$. Consequently, the stability of the system is proved.

In what follows, the stability of the proposed MF-iPINLFOSMC strategy will be discussed.

Proof. In order to guarantee the stability of the proposed MF-iPI-NLFOSMC strategy, the Lyapunov function for the proposed MF-iPI-NLFOSMC strategy is defined as

$$
V=\frac{1}{2} s_{\text {NLFOSM }}(t)^{2}>0 .
$$

Taking the derivatives of both sides of (43), the following equation is derived:

$$
\begin{aligned}
& \dot{V}=s_{\mathrm{NLFOSM}}(t) \cdot \dot{s}_{\mathrm{NLFOSM}}(t) \\
& =s_{\text {NLFOSM }}(t)\left(K_{n l f p} D_{t}^{1} f a l(e(t))+K_{n l f i} D_{t}^{u} f a l(e(t))+K_{n l f d} D_{t}^{1+\varepsilon} f a l(e(t))\right) \\
& =s_{\text {NLFOSM }}(t)\left(\begin{array}{l}
K_{n l f p} \widetilde{\alpha}|e|^{\tilde{\alpha}-1}\left(-a u_{2}-K_{p} e(t)-K_{i} \int e(t) \mathrm{d} t-\Delta \mathrm{d}(t)\right)+K_{n l f i} D_{t}^{u} f a l(e(t))+K_{n l f} D_{t}^{1+\varepsilon} \text { fal }(e(t)) \quad \text { if }|e(t)|>\tilde{\delta} \\
\left(\frac{K_{n l f p}}{\tilde{\delta}^{1-\tilde{\alpha}}}\right)\left(-a u_{2}-K_{p} e(t)-K_{i} \int e(t) \mathrm{d} t-\Delta \mathrm{d}(t)\right)+K_{n l f i} D_{t}^{u} \mathrm{fal}(e(t))+K_{n l f d} D_{t}^{1+\varepsilon} f a l(e(t)) \quad \text { if }|e(t)| \leq \tilde{\delta}
\end{array}\right) \\
& =s_{\mathrm{NLFOSM}}(t)\left(\begin{array}{c}
-K_{n l f p} \widetilde{\alpha}|e|^{\tilde{\alpha}-1} \eta \operatorname{sign}\left(s_{\mathrm{NLFOSM}}(t)\right)-K_{n l f p} \widetilde{\alpha}|e|^{\tilde{\alpha}-1} \Delta d(t) \quad \text { if }|e(t)|>\tilde{\delta} \\
-\left(\frac{K_{n l f p}}{\tilde{\delta}^{1-\widetilde{\alpha}}}\right) \eta \operatorname{sign}\left(s_{\mathrm{NLFOSM}}(t)\right)-\left(\frac{K_{n l f p}}{\widetilde{\delta}^{1-\tilde{\alpha}}}\right) \Delta \mathrm{d}(t) \quad \text { if }|e(t)| \leq \tilde{\delta}
\end{array}\right) \\
& =\left\{\begin{array}{l}
-K_{n l f p} \widetilde{\alpha}|e|^{\tilde{\alpha}-1} \eta\left|s_{\mathrm{NLFOSM}}(t)\right|-s_{\mathrm{NLFOSM}}(t) K_{n l f p} \widetilde{\alpha}|e|^{\tilde{\alpha}-1} \Delta \mathrm{d}(t) \quad \text { if }|e(t)|>\widetilde{\delta} \\
-\left(\frac{K_{n l f p}}{\tilde{\delta}^{1-\tilde{\alpha}}}\right) \eta\left|s_{\mathrm{NLFOSM}}(t)\right|-s_{\mathrm{NLFOSM}}(t)\left(\frac{K_{n l f p}}{\tilde{\delta}^{1-\tilde{\alpha}}}\right) \Delta \mathrm{d}(t) \quad \text { if }|e(t)| \leq \widetilde{\delta}
\end{array}\right. \\
& \leq-\widetilde{K} \eta\left|s_{\text {NLFOSM }}(t)\right|+\left|s_{\text {NLFOSM }}(t)\right| \widetilde{K}|\Delta \mathrm{d}(t)|,
\end{aligned}
$$

where

$$
\widetilde{K}=\left\{\begin{array}{l}
K_{n l f p} \widetilde{\alpha}|e(t)|^{\tilde{\alpha}-1} \quad \text { if }|e(t)|>\widetilde{\delta}, \\
\left(\frac{K_{n l f p}}{\widetilde{\delta}^{1-\widetilde{\alpha}}}\right) \text { if }|e(t)| \leq \widetilde{\delta},
\end{array}\right.
$$

and we know that the error $e(t)$ of the real systems cannot be completely eliminated at the reaching stage, so $\widetilde{K}>0$ is satisfied.

According to Assumption 1 and when $\eta>\Psi$ is satisfied, we can get $\dot{V}<0$. Consequently, the stability of the system is proved. In what follows, inspired by the above studies $[55,56]$, the stability of the proposed MF-iPI-ST-NLFOSMC strategy will be discussed.

Proof. In order to guarantee the stability of the proposed MF-iPI-ST-NLFOSMC strategy, the Lyapunov function for the proposed MF-iPI-ST-NLFOSMC strategy is defined as

$$
V=\frac{1}{2} s_{\text {NLFOSM }}(t)^{2}>0 .
$$

Taking the derivatives of both sides of equation (26), the following equation can be obtained: 


$$
\begin{aligned}
\dot{s}_{\text {NLFOSM }}(t)= & K_{n l f p} D_{t}^{1} \mathrm{fal}(e(t))+K_{n l f i} D_{t}^{u} \mathrm{fal}(e(t))+K_{n l f d} D_{t}^{1+\varepsilon} \mathrm{fal}(e(t)), \\
& =\left\{\begin{array}{l}
K_{n l f p} \widetilde{\alpha}|e|^{\tilde{\alpha}-1} \dot{e}(t)+K_{n l f i} D_{t}^{u} f a l(e(t))+K_{n l f d} D_{t}^{1+\varepsilon} f a l(e(t)) \quad \text { if }|e(t)|>\widetilde{\delta}, \\
\left(\frac{K_{\mathrm{nlfp}}}{\tilde{\delta}^{1-\widetilde{\alpha}}}\right) \dot{e}(t)+K_{n l f i} D_{t}^{u} f a l(e(t))+K_{n l f d} D_{t}^{1+\varepsilon} f a l(e(t)) \quad \text { if }|e(t)| \leq \widetilde{\delta}, \\
= \\
\quad\left(\frac{K_{\mathrm{nlfp}}}{\widetilde{\delta}^{1-\tilde{\alpha}}}\right)\left(-a u_{2}-K_{p} e(t)-K_{i} \int e(t) \mathrm{d} t-\Delta \mathrm{d}(t)\right)+K_{n l f i} D_{t}^{u} f a l(e(t)) \\
K_{\mathrm{nlfp}} \widetilde{\alpha}|e|^{\tilde{\alpha}-1}\left(-a u_{2}-K_{p} e(t)-K_{i} \int e(t) \mathrm{d} t-\Delta \mathrm{d}(t)\right)+K_{n l f i} D_{t}^{u} f a l(e(t))+K_{n l f d} D_{t}^{1+\varepsilon} f a l(e(t)) \quad \text { if }|e(t)|>\widetilde{\delta}, \\
+K_{n l f d} D_{t}^{1+\varepsilon} f a l(e(t)) \quad|e(t)| \leq \widetilde{\delta} .
\end{array}\right.
\end{aligned}
$$

By substituting (33) into (47), we obtain the following:

$$
\begin{aligned}
\dot{s}_{\text {NLFOSM }}(t)= & -\widetilde{K} k_{1}\left|s_{\text {NLFOSM }}(t)\right|^{(1 / 2)} \operatorname{sign}\left(s_{\text {NLFOSM }}(t)\right) \\
& -\widetilde{K} k_{2} \int \operatorname{sign}\left(s_{\text {NLFOSM }}(t)\right) \mathrm{d} t-\widetilde{K} \Delta \mathrm{d}(t) .
\end{aligned}
$$

According to (46) and (48), the gains of $k_{1}$ and $k_{2}$ are set to be larger enough, and the following system stability can be satisfied:

$$
\begin{aligned}
\dot{V}= & s_{\text {NLFOSM }}(t) \cdot \dot{s}_{\text {NLFOSM }}(t) \\
= & -\widetilde{K} s_{\text {NLFOSM }}(t)\left(k_{1}\left|s_{\text {NLFOSM }}(t)\right|^{(1 / 2)} \operatorname{sign}\left(s_{\text {NLFOSM }}(t)\right)\right. \\
& +k_{2} \int \operatorname{sign}\left(s_{\text {NLFOSM }}(t) \mathrm{d} t+\Delta \mathrm{d}(t)\right)<0,
\end{aligned}
$$

where

$$
s_{\mathrm{NLFOSM}}(t) \cdot \operatorname{sign}\left(s_{\mathrm{NLFOSM}}(t)\right)>0 \text {. }
$$

We can conclude that the system is stable. A detailed description can be found in references $[55,56]$.

\section{Comparative Simulations}

For the sake of demonstrating the effectiveness of the proposed MF-iPI-ST-NLFOSMC strategy, we set up the field-oriented control of the PMSM speed regulation system based on different control strategies at the Matlab (R2014a)/ Simulink. The traditional MF-iPI-SMC, the MF-iPIFOSMC, and the MF-iPI-NLFOSMC strategies are compared with the control strategy proposed in this study.

Table 1 shows the parameters of the PMSM. Tables 2-5 show the parameters of the traditional MF-iPI-SMC, the MF-iPI-FOSMC, the MF-iPI-NLFOSMC, and the MF-iPIST-NLFOSMC strategies, respectively. In order to better verify the superiority of the proposed control strategy, some parameters in various control strategies are set to the same, which can better eliminate the influence of parameter setting on the comparison results.
4.1. Case I: Comparison of Speed Response Curves. In order to compare the control performance of various controllers, the reference speed is set to $30 \mathrm{r} / \mathrm{s}$ and the simulation time is set to $2 \mathrm{~s}$. Figures 4 and 5 demonstrate the response curves of the speed under the traditional MF-iPI-SMC, the MF-iPIFOSMC, the MF-iPI-NLFOSMC, and the MF-iPI-STNLFOSMC with no load and load, respectively. In order to compare more accurately, the stable simulation data from $0.5 \mathrm{~s}$ to $2 \mathrm{~s}$ are used to perform the following calculations:

$$
\begin{aligned}
\text { root }- \text { mean }- \text { square error }(\mathrm{RMSE}) & =\sqrt{\frac{\sum_{1}^{N} e_{i}(t)^{2}}{N},} \\
\text { maximum absolute error }(\mathrm{MAE}) & =\operatorname{Max}\left|e_{i}(t)\right| .
\end{aligned}
$$

Figures 6 and 7 demonstrate the RMSE and MAE under the traditional MF-iPI-SMC, the MF-iPI-FOSMC, the MFiPI-NLFOSMC, and the MF-iPI-ST-NLFOSMC with no load and load, respectively. As can be seen from Figures 4 and 5, the reaching time under the proposed MF-iPI-STNLFOSMC is shorter than the reaching times under the other control strategies. It reveals that the proposed MF-iPIST-NLFOSMC strategy has the best dynamic characteristics. Figures 6 and 7 clearly show that the RMSE and MAE of the proposed MF-iPI-ST-NLFOSMC strategy are minimal, which indicates that the static characteristic of the proposed MF-iPI-ST-NLFOSMC strategy is the best.

4.2. Case II: Comparison of Resistance to External Disturbances. A sudden change in external load is used to verify the robustness of the proposed MF-iPI-STNLFOSMC strategy. The reference speed is set to $30 \mathrm{r} / \mathrm{s}$, and the simulation time is set to $0.7 \mathrm{~s}$. The external load suddenly becomes $0.6 \mathrm{~N} \times \mathrm{m}$ from $0 \mathrm{~N} \times \mathrm{m}$ at $0.3 \mathrm{~s}$. The external load suddenly becomes $0 \mathrm{~N} \times \mathrm{m}$ from $0.6 \mathrm{~N} \times \mathrm{m}$ at $0.5 \mathrm{~s}$. Figure 8 compares the speed response curves under different control strategies with sudden load change. Table 6 shows the comparative results of the sudden load change under different control strategies, including speed perturbation and 
TABle 1: Parameters of the PMSM.

\begin{tabular}{lcccc}
\hline$L$ & $\phi_{f}$ & $J$ & $B$ & $p_{n}$ \\
\hline $8.5 \mathrm{mH}$ & $0.175 \mathrm{~Wb}$ & $0.003 \mathrm{~kg} \cdot \mathrm{m}^{2}$ & $0.008 \mathrm{~N} \cdot \mathrm{m} \cdot \mathrm{s} / \mathrm{rad}$ & 4 \\
\hline
\end{tabular}

TABLe 2: Key parameters of the traditional MF-iPI-SMC.

\begin{tabular}{ccccccccc}
\hline \multicolumn{4}{c}{ MF-iPI-SMC } & \multicolumn{2}{c}{ LESO } \\
$K_{p}$ & $K_{i}$ & $a$ & $\eta$ & $\eta_{1}$ & $\eta_{2}$ & $\beta_{1}$ & $\beta_{2}$ \\
\hline 1 & 0.3 & 1000 & 400 & 0.1 & 1 & 2000 & 1000000 \\
\hline
\end{tabular}

TABle 3: Key parameters of the MF-iPI-FOSMC.

\begin{tabular}{lccccccc}
\hline \multicolumn{9}{c}{ MF-iPI-FOSMC } & \multicolumn{2}{c}{ LESO } \\
$K_{p}$ & $K_{i}$ & $a$ & $\eta$ & $K_{\mathrm{fp}}$ & $K_{\mathrm{fi}}$ & $\beta_{1}$ & $\beta_{2}$ \\
\hline 1 & 0.3 & 1000 & 400 & 0.3 & 0.3 & 2000 & 1000000 \\
$K_{f d}$ & $\varepsilon$ & $u$ & & & & \\
0.3 & 0.01 & 0.99 & & & & \\
\hline
\end{tabular}

TABLE 4: Key parameters of the MF-iPI-NLFOSMC.

\begin{tabular}{lccccccc}
\hline & \multicolumn{9}{c}{ MF-iPI-NLFOSMC } & \multicolumn{2}{c}{ LESO } \\
$K_{p}$ & $K_{i}$ & $a$ & $\eta$ & $K_{\text {nlfp }}$ & $K_{\text {nlfi }}$ & $\beta_{1}$ & $\beta_{2}$ \\
\hline 1 & 0.3 & 1000 & 400 & 0.3 & 0.3 & 2000 & 1000000 \\
$K_{n l f d}$ & $\varepsilon$ & $u$ & $\tilde{\alpha}$ & 0.1 & & \\
0.3 & 0.01 & 0.99 & 0.25 & 0.1 & & \\
\hline
\end{tabular}

TABLE 5: Key parameters of the MF-iPI-ST-NLFOSMC.

\begin{tabular}{lccccccc}
\hline & \multicolumn{3}{c}{ MF-iPI-ST-NLFOSMC } & \multicolumn{3}{c}{ LESO } \\
$K_{p}$ & $K_{i}$ & $a$ & $K_{\text {nlfd }}$ & $K_{\text {nlf }}$ & $K_{\text {nlfi }}$ & $\beta_{1}$ & $\beta_{2}$ \\
\hline 1 & 0.3 & 1000 & 0.3 & 0.3 & 0.3 & 2000 & 1000000 \\
$\varepsilon$ & $u$ & $\widetilde{\sigma}$ & 0.1 & $k_{1}$ & $k_{2}$ & & 1000 \\
0.01 & 0.99 & 0.25 & 0.1000 & 100 & \\
\hline
\end{tabular}

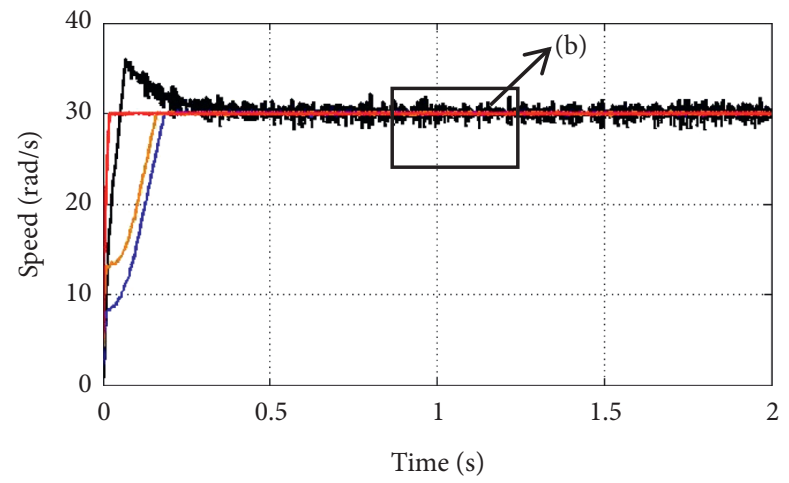

MF-iPI-ST-NLFOSMC - MF-iPI-FOSMC
MF-iPI-NLFOSMC - Traditional MF-iPI-SMC

(a)

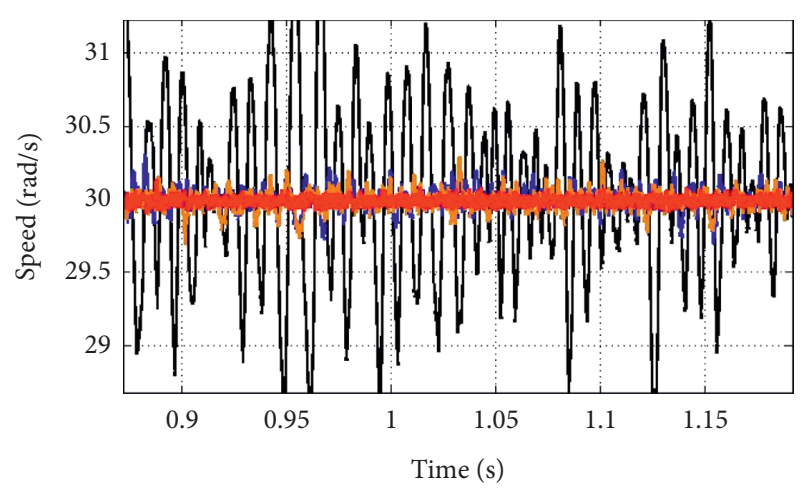

MF-iPI-ST-NLFOSMC - MF-iPI-FOSMC
MF-iPI-NLFOSMC - Traditional MF-iPI-SMC

(b)

FIgURE 4: (a) Speed response curves under the traditional MF-iPI-SMC, the MF-iPI-FOSMC, the MF-iPI-NLFOSMC, and the MF-iPI-STNLFOSMC without load. (b) Magnification pictures of the speed response curves under the control strategies without load. 


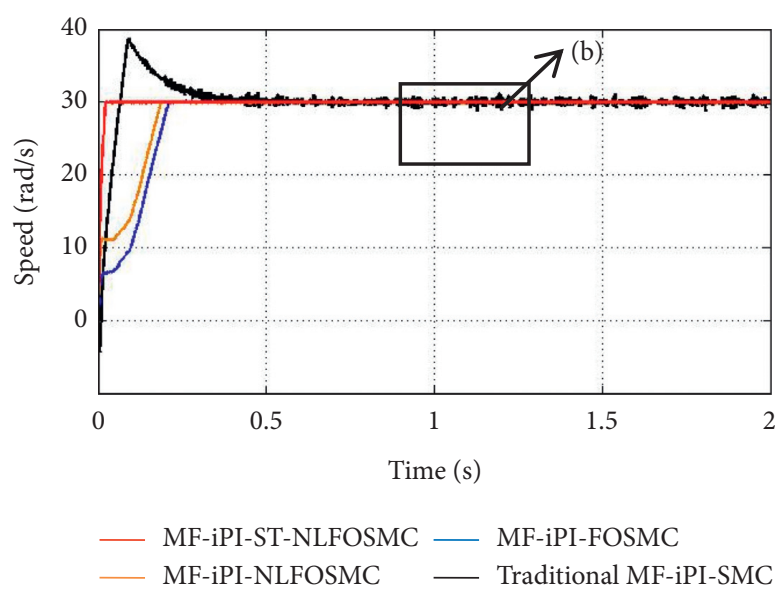

(a)

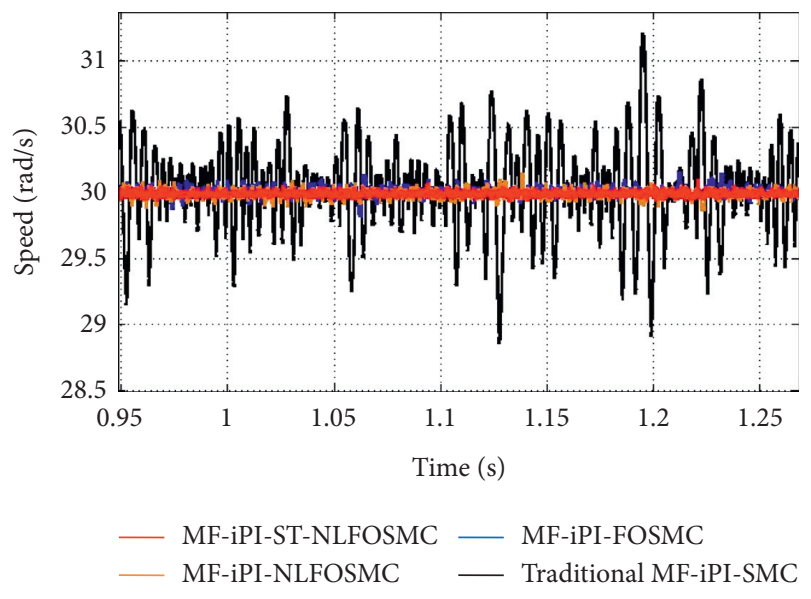

(b)

FIgURE 5: (a) Speed response curves under the traditional MF-iPI-SMC, the MF-iPI-FOSMC, the MF-iPI-NLFOSMC, and the MF-iPI-STNLFOSMC with load. (b) Magnification pictures of the speed response curves under the control strategies with load.

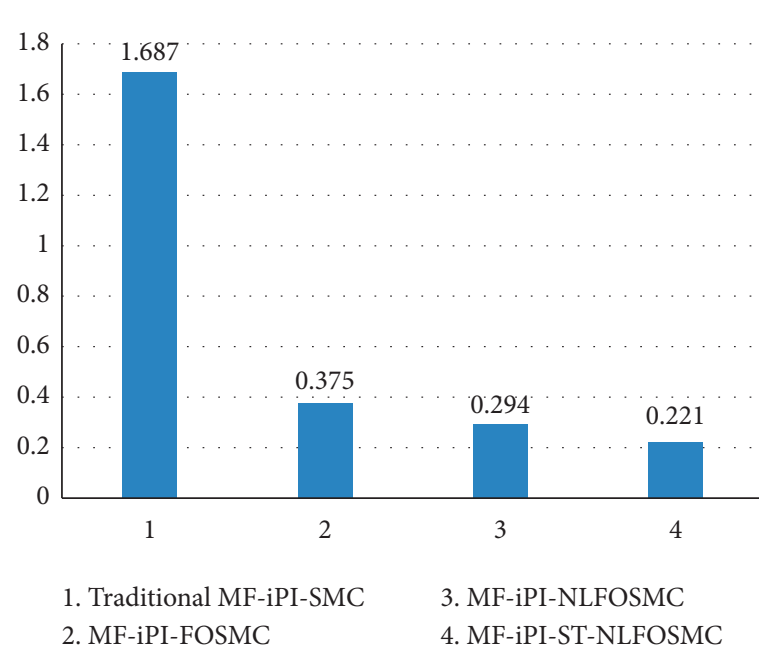

(a)

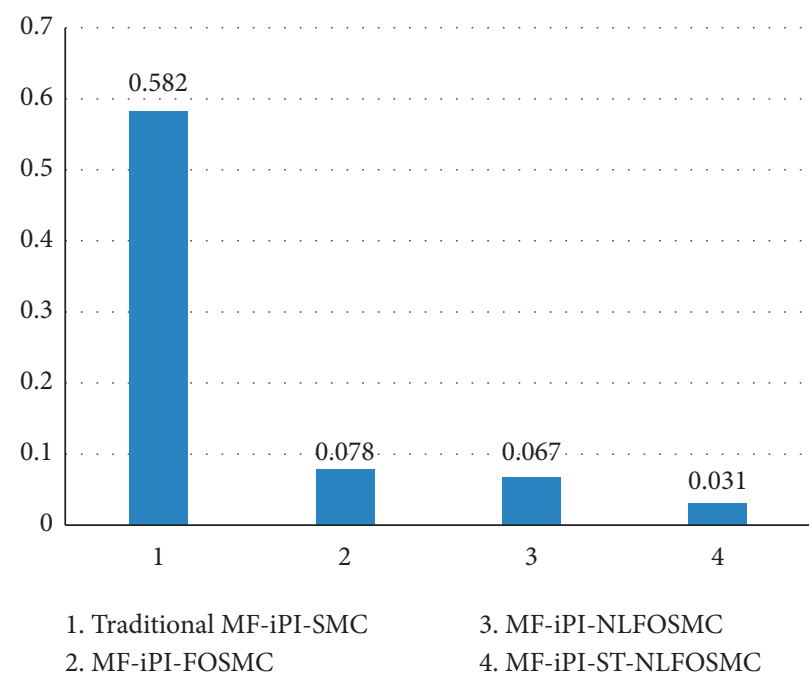

(b)

Figure 6: (a) Maximum absolute errors under the traditional MF-iPI-SMC, the MF-iPI-FOSMC, the MF-iPI-NLFOSMC, and the MF-iPIST-NLFOSMC without load. (b) Root-mean-square errors under the traditional MF-iPI-SMC, the MF-iPI-FOSMC, the MF-iPINLFOSMC, and the MF-iPI-ST-NLFOSMC without load.

speed recovery time. It can be seen from Figure 8 and Table 6 that all control strategies are affected by external load disturbance. The fluctuation impact rate and recovery time under the proposed MF-iPI-ST-NLFOSMC strategy are smaller than those under the other control methods, which reveal the proposed MF-iPI-ST-NLFOSMC strategy has better robustness compared with the other control methods.

4.3. Case III: Comparison of Antinoise Interference. A noise interference is added to the given speed to verify the antinoise performance of the proposed MF-iPI-ST-NLFOSMC strategy. The reference speed with the noise interference is shown in Figure 9(a). It can be seen from Figure 9(b) and Figure 9(c) that all control strategies are affected by the noise interference. The fluctuation impact rate under the proposed
MF-iPI-ST-NLFOSMC strategy is smaller than those under the other control methods, which reveals that the proposed MF-iPI-ST-NLFOSMC strategy has better antinoise interference ability compared with the other control methods.

\section{Conclusions and Future Work}

A novel MF-iPI-ST-NLFOSMC strategy based on the ultralocal model is proposed for the control of PMSM speed regulation system. The LESO is used to estimate the unknown terms of the ultralocal model of PMSM. The stability of the closed-loop system is proved by the Lyapunov stability theorem and fractional-order theory. The proposed MF-iPIST-NLFOSMC strategy consists of the model-free STNLFOSMC strategy, the model-free iPI control strategy, and 


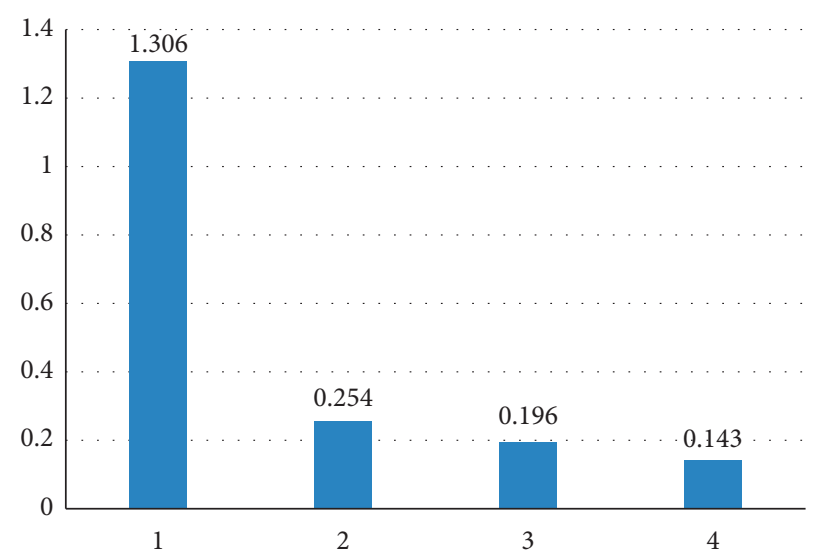

1. Traditional MF-iPI-SMC 2. MF-iPI-FOSMC

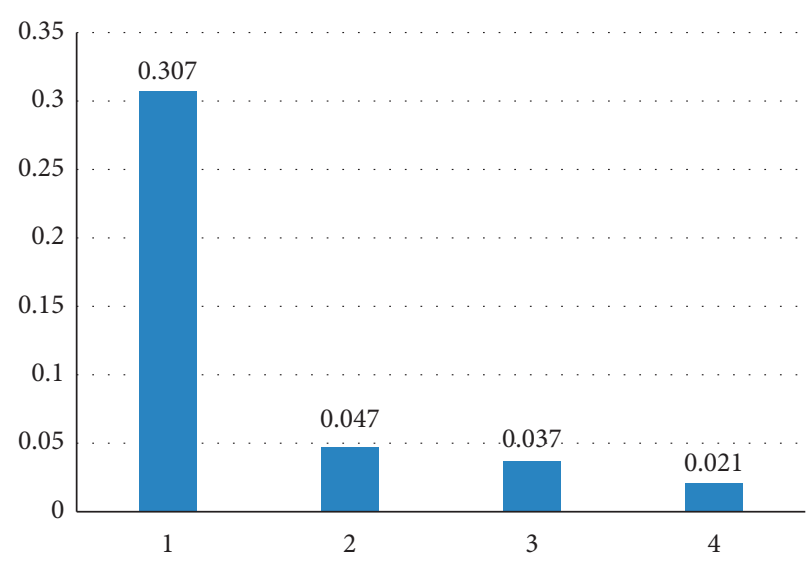

1. Traditional MF-iPI-SMC 2. MF-iPI-FOSMC
3. MF-iPI-NLFOSMC

4. MF-iPI-ST-NLFOSMC

(a)

(b)

FIGURE 7: (a) Maximum absolute errors under the traditional MF-iPI-SMC, the MF-iPI-FOSMC, the MF-iPI-NLFOSMC, and the MF-iPIST-NLFOSMC with load. (b) Root-mean-square errors under the traditional MF-iPI-SMC, the MF-iPI-FOSMC, the MF-iPI-NLFOSMC, and the MF-iPI-ST-NLFOSMC with load.

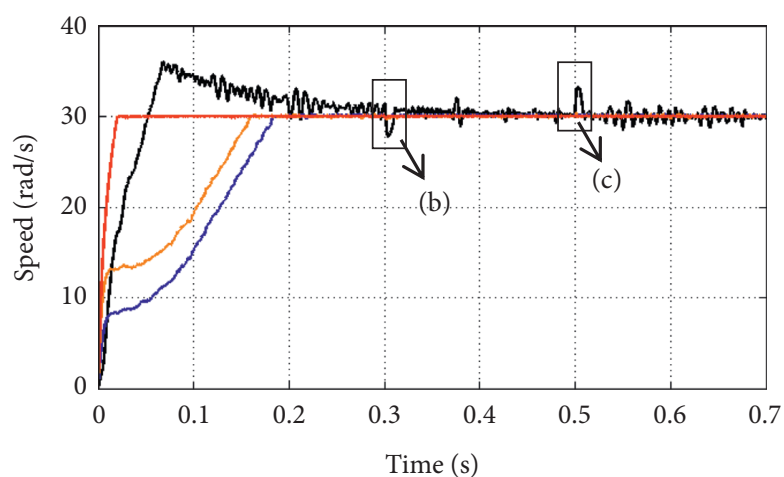

MF-iPI-ST-NLFOSMC - MF-iPI-FOSMC
MF-iPI-NLFOSMC $\quad$ Traditional MF-iPI-SMC

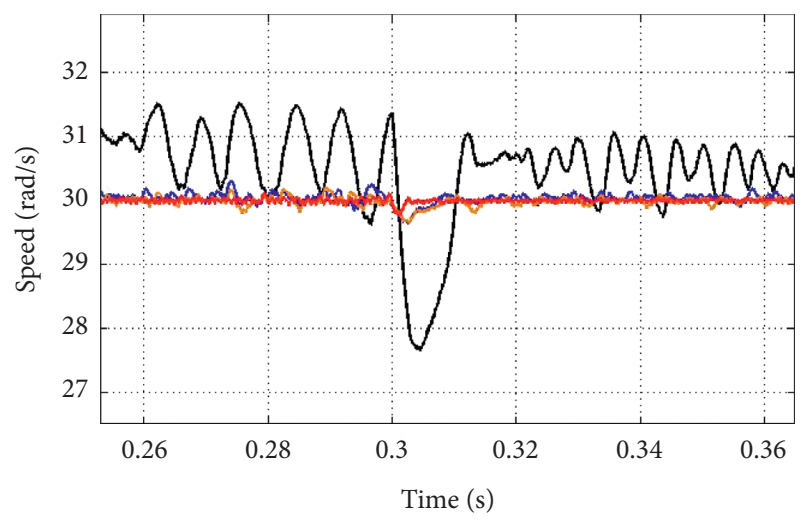

- MF-iPI-ST-NLFOSMC — MF-iPI-FOSMC — MF-iPI-NLFOSMC — Traditional MF-iPI-SMC

(b)

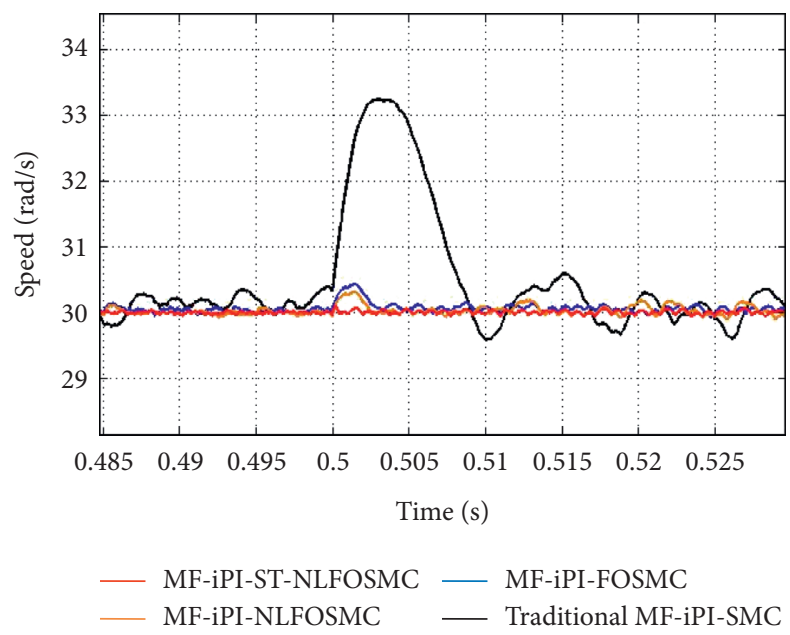

(c)

Figure 8: (a) Speed response curves when the external load changes suddenly. (b) Partial enlarged views of speed response curves when the external load becomes $0.7 \mathrm{~N} \times m$ from $0 \mathrm{~N} \times m$ at $0.3 \mathrm{~s}$. (c) Partial enlarged views of speed response curves when the external load becomes 0 $\mathrm{N} \times m$ from $0.7 \mathrm{~N} \times m$ at $0.5 \mathrm{~s}$. 
TABLE 6: The comparison of resistance to external disturbances.

\begin{tabular}{lcc}
\hline Control strategies & $\begin{array}{c}\text { Sudden increase in external load at } 0.3 \mathrm{~s} \\
\text { Impact rate of fluctuation (\%) }\end{array}$ & $\begin{array}{c}\text { Sudden decrease in external load at } 0.5 \mathrm{~s} \\
\text { Impact rate of fluctuation (\%) }\end{array}$ \\
\hline Traditional MF-iPI-SMC & 7.81 & 8.17 \\
MF-iPI-FOSMC & 1.21 & 1.47 \\
MF-iPI-NLFOSMC & 1.24 & 1.07 \\
MF-iPI-ST-NLFOSMC & 0.83 & 0.69 \\
\hline
\end{tabular}

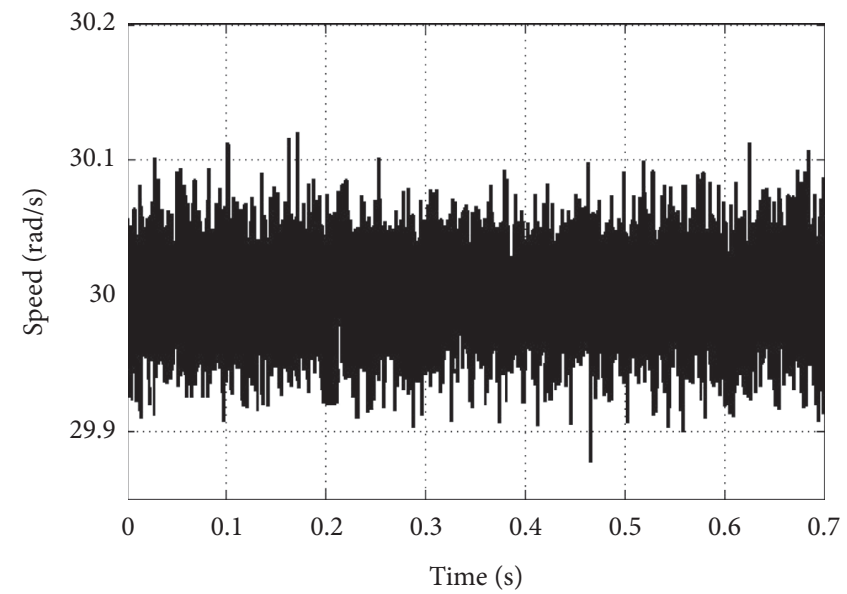

(a)

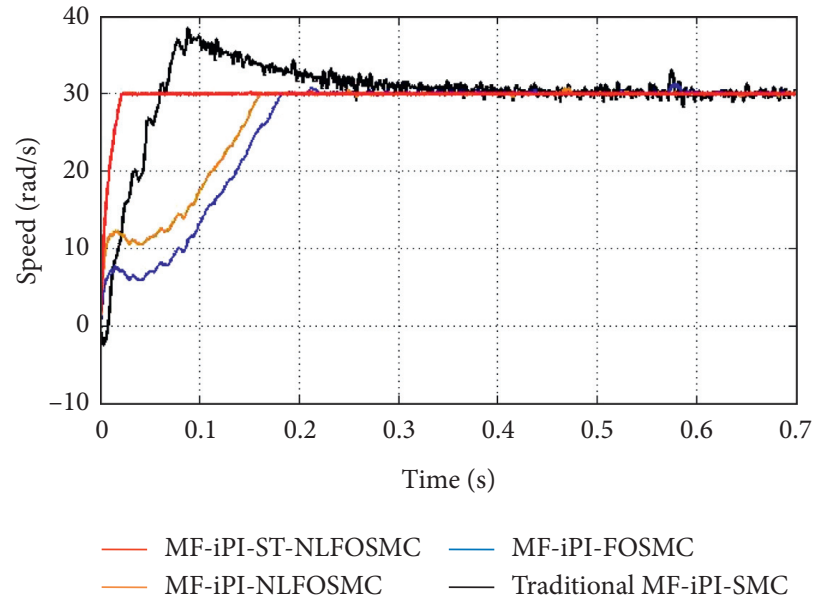

(b)

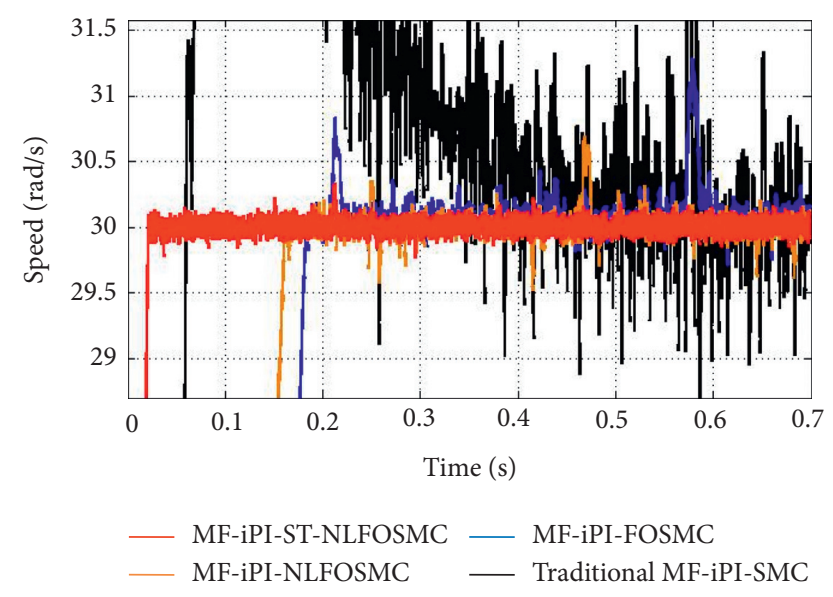

(c)

Figure 9: (a) Given speed with noise interference. (b) Speed response curves with the noise interference. (c) Partial enlarged views of (b).

the LESO, which has excellent performance. It is found that simulation experiments and comparison results demonstrate that the proposed control algorithm not only achieves better stability and dynamic properties but also has stronger robustness to external disturbance compared with LESObased other model-free control methods.

The use of the model-free ST-NLFOSMC strategy, the model-free iPI control strategy, and the LESO in this paper leads to the increase of control method parameters, which increases the complexity of the method. In the future work, we will select an optimization algorithm to complete the selection of the parameters, which will further improve the performance of the control method proposed in this paper.

\section{Data Availability}

The data used to support the findings of this study are included within the article.

\section{Conflicts of Interest}

The authors declare no conflicts of interest.

\section{Acknowledgments}

This work was supported by the National Natural Science Foundation of China under Grant no. 61703202 and Key 
Research Development Project of Jiangsu Province under Grant BE2017164.

\section{References}

[1] E. M. Tsampouris, M. E. Beniakar, and A. G. Kladas, "Geometry optimization of PMSMs comparing full and fractional pitch winding configurations for aerospace actuation applications," IEEE Transactions on Magnetics, vol. 48, no. 289, pp. 943-946, 2012.

[2] H. Li, T. Chen, and H. Yao, "Mechanism, diagnosis and development of demagnetization fault for PMSM in electric vehicle," Transactions of China Electrotechnical Society, vol. 28, no. 8, pp. 276-284, 2013.

[3] H. Khayamy, M. Khayamy, and O. Okoye, "Adaptive RBF network based direct voltage control for interior PMSM based vehicles," IEEE Transactions on Vehicular Technology, vol. 67, no. 7, pp. 5740-5749, 2018.

[4] B. Kang and C. Liaw, "A robust hysteresis current-controlled PWM inverter for linear PMSM driven magnetic suspended positioning system," IEEE Transactions on Industrial Electronics, vol. 48, no. 5, pp. 956-967, 2001.

[5] M. Preindl and S. Bolognani, "Model predictive direct speed control with finite control set of PMSM drive systems," IEEE Transactions on Power Electronics, vol. 28, no. 2, pp. 10071015, 2013.

[6] S. Li, H. Liu, and S. Ding, "A speed control for a PMSM using finite-time feedback control and disturbance compensation," Transactions of the Institute of Measurement and Control, vol. 32, no. 2, pp. 170-187, 2010.

[7] S. Wu, J. Zhang, and B. Chai, "A robust backstepping sensorless control for interior permanent magnet synchronous motor using a super-twisting based torque observer," Asian Journal of Control, vol. 21, no. 1, pp. 172-183, 2019.

[8] S. Kim, J. Lee, and K. Lee, "Singularity-free adaptive speed tracking control for uncertain permanent magnet synchronous motor," IEEE Transactions on Power Electronics, vol. 31, no. 2, pp. 7065-7076, 2016.

[9] W. Wang, H. Shen, L. Hou, and H. Gu, "Horobust control of permanent magnet synchronous motor based on PCHD," IEEE Access, vol. 7, pp. 49150-49156, 2019.

[10] S. Li, J. Li, and Y. Shi, "An RBFNN-based direct inverse controller for PMSM with disturbances," Complexity, vol. 2018, Article ID 4034320, 13 pages, 2018.

[11] Y.-Q. Wang, Y.-T. Feng, X.-G. Zhang, J. Liang, and X. Cheng, "A new reaching law for anti-disturbance sliding-mode control of PMSM speed regulation system," IEEE Access, vol. 7, pp. 49150-49156, 2019.

[12] S. Mobayen and F. Tchier, "Synchronization of a class of uncertain chaotic systems with Lipschitz nonlinearities using state-feedback control design: A matrix inequality approach," Asian Journal of Control, vol. 20, no. 1, pp. 1-15, 2018.

[13] M. Fliess and C. Join, "Intelligent PID controllers," in Proceedings of the the 16th Mediterranean Conference on Control and Automation, pp. 25-27, Ajaccio, France, June 2008.

[14] J. T. Agee, S. Kizir, and Z. Bingul, "Intelligent proportionalintegral (iPI) control of a single link flexible joint manipulator," Journal of Vibration and Control, vol. 21, no. 11, pp. 2273-2288, 2015.

[15] M. Gheisarnejad, H. Farsizadeh, and M. H. Khooban, "A novel non-linear deep reinforcement learning controller for DC/DC power buck converters," IEEE Transactions on Industrial Electronics, p. 1, 2020.
[16] J. T. Agee, Z. Bingul, and S. Kizir, “Tip trajectory control of a flexible-link manipulator using an intelligent proportional integral (iPI) controller," Transactions of the Institute of Measurement and Control, vol. 36, no. 5, pp. 673-682, 2014.

[17] Y. Liu, W. Yan, D. Xu, W. Yang, and W. Zhang, "Direct torque control of PMSM based on model free iPI controller," in Proceedings of the IEEE 7th Data Driven Control and Learning Systems Conference, Enshi, China, May 2018.

[18] S. Mobayen, M. Yazdanpanah, and V. Majd, "A finite-time tracker for nonholonomic systems using recursive singularityfree FTSM," in Proceedings of the 2011 American Control Conference, San Francisco, CA, USA, June 2011.

[19] S. Mobayen and F. Tchier, "Nonsingular fast terminal sliding mode stabilizer for a class of uncertain nonlinear systems based on disturbance observer," Scientia Iranica, vol. 24, no. 9, 2017.

[20] Y. Wang, S. Li, D. Wang, F. Ju, B. Chen, and H. Wu, “Adaptive time-delay control for cable-driven manipulators with enhanced nonsingular fast terminal sliding mode," IEEE Transactions on Industrial Electronics, p. 1, 2020.

[21] Y. Wang, L. Gu, Y. Xu, and X. Cao, "Practical tracking control of robot manipulators with continuous fractional-order nonsingular terminal sliding mode," IEEE Transactions on Industrial Electronics, vol. 63, no. 10, pp. 6194-6204, 2016.

[22] S. Li, H. Wang, A. Aitouch, and J. Klein, "Direct power control of DFIG wind turbine systems based on an intelligent proportional-integral sliding mode control," ISA Transactions, vol. 64, pp. 431-439, 2016.

[23] M. Roohi, C. Zhang, and Y. Chen, "Adaptive model-free synchronization of different fractional-order neural networks with an application in cryptography," Nonlinear Dynamics, vol. 100, no. 4, pp. 3979-4001, 2020.

[24] M. P. Aghababa, M. Roohi, and A. R. Haghighi, "Stabilisation of unknown fractional-order chaotic systems: an adaptive switching control strategy with application to power systems," IET Generation, Transmission \& Distribution, vol. 9, no. 14, pp. 1883-1893, 2015.

[25] H.-P. Ren, X. Wang, J.-T. Fan, and O. Kaynak, "Fractional order sliding mode control of a pneumatic position servo system," Journal of the Franklin Institute, vol. 356, no. 12, pp. 6160-6174, 2019.

[26] X. Rui, W. Yin, Y. Dong, L. Lin, and X. Wu, "Fractional-order sliding mode control for hybrid drive wind power generation system with disturbances in the grid," Wind Energy, vol. 22, no. 1, pp. 49-64, 2019.

[27] J. Wang, Y. Zhou, Y. Bao, H. Kim, and M. Lee, "Trajectory tracking control using Fractional-order terminal sliding mode control with sliding perturbation observer for a 7-DOF robot manipulator," IEEE/ASME Transactions on Mechatronics, vol. 25, no. 4, pp. 1886-1893, 2020.

[28] P. Li, J. Wang, L. Xiong, S. Huang, Z. Wang, and M. Ma, "SSCI mitigation of grid-connected DFIG wind turbines with fractional-order sliding mode controller," Wind Energy, vol. 23, no. 7, pp. 1564-1577, 2020.

[29] H.-R. Li, Z.-B. Jiang, and N. Kang, "Sliding mode disturbance observer-based fractional second-order nonsingular terminal sliding mode control for PMSM position regulation system," Mathematical Problems in Engineering, vol. 2015, Article ID 370904, 14 pages, 2015.

[30] J.-T. Fei, Z. Wang, and X. Liang, "Robust adaptive fractional fast terminal sliding mode controller for microgyroscope," Complexity, vol. 2020, Article ID 8542961, 18 pages, 2020.

[31] Z. Esfahani, M. Roohi, M. Gheisarnejad, T. Dragičević, and M.-H. Khooban, "Optimal non-integer sliding mode control 
for frequency regulation in stand-alone modern power grids," Applied Sciences, vol. 9, no. 16, p. 3411, 2019.

[32] M. Roohi, M.-H. Khooban, Z. Esfahani, M. P. Aghababa, and T. Dragicevic, "A switching sliding mode control technique for chaos suppression of fractional-order complex systems," Transactions of the Institute of Measurement and Control, vol. 41, no. 10, pp. 2932-2946, 2019.

[33] J. Song, L. Wang, G. Cai, and X. Qi, "Nonlinear fractional order proportion-integral-derivative active disturbance rejection control method design for hypersonic vehicle attitude control," Acta Astronautica, vol. 111, pp. 160-169, 2015.

[34] M. Farshad and M. Nafiseh, "Introduction to the nonlinear $P I^{\lambda} D^{u}$ control," in Proceedings of the 2011 IEEE International Conference on Control System, Computing and Engineering, pp. 25-27, Penang, Malaysia, November 2011.

[35] L. Shi, X. Miao, and H. Wang, "An improved nonlinear proportional integral-differential controller combined with fractional operator and symbolic adaptation algorithm," Transactions of the Institute of Measurement and Control, vol. 42, no. 5, 2019.

[36] P. Gao, G. Zhang, H. Ouyang, and L. Mei, "A sliding mode control with nonlinear fractional order PID sliding surface for the speed operation of surface-mounted PMSM drives based on an extended state observer," Mathematical Problems in Engineering, vol. 2019, Article ID 7130232, 13 pages, 2019.

[37] P. Gao, G. Zhang, and X. Lv, "A novel compound nonlinear state error feedback super-twisting fractional-order sliding mode control of PMSM speed regulation system based on extended state observer," Mathematical Problems in Engineering, vol. 2020, Article ID 1843598, 15 pages, 2020.

[38] P. Gao, G. Zhang, H. Ouyang, and L. Mei, "An adaptive super twisting nonlinear fractional order PID sliding mode control of permanent magnet synchronous motor speed regulation system based on extended state observer," IEEE Access, vol. 8, pp. 53498-53510, 2020.

[39] A. Levant, "Robust exact differentiation via sliding mode technique," Automatica, vol. 34, no. 3, pp. 379-384, 1998.

[40] Z. Gao, "Active disturbance rejection control: A paradigm shift in feedback control system design," in Proceedings of the 2006 American Control Conference, pp. 2399-2405, Minneapolis, MN, USA, June 2006.

[41] Z. Gao, Y. Huang, and J. Han, "An alternative paradigm for control system design," in Proceedings of the 40th IEEE Conference on Decision and Control, Orlando, FL, USA, December 2001.

[42] Q. Wu, L. Yu, Y. Wang, and W. Zhang, "LESO-based position synchronization control for networked multi-axis servo systems with time-varying delay," IEEE-CAA Journal of Automatica Sinica, vol. 7, no. 4, pp. 1116-1123, 2020.

[43] K. Miao, X. Shi, and W. A. Zhang, "Attack signal estimation for intrusion detection in industrial control system," Computers \& Security, vol. 96, Article ID 101926.

[44] Y. W. Wang, W. A. Zhang, and L. Yu, "A linear active disturbance rejection control approach to position synchronization control for networked interconnected motion system," IEEE Transactions on Control of Network Systems, p. 1, 2020.

[45] K. Gao, J. Song, X. Wang, and H.-F. Li, "Fractional-order proportional-integral-derivative linear active disturbance rejection control design and parameter optimization for hypersonic vehicles with actuator faults," Tsinghua Science and Technology, vol. 26, no. 1, pp. 9-23, 2021.

[46] I. Podlubny, Fractional Differential Equations: An Introduction to Fractional Derivatives, Fractional Differential
Equations, to Methods of their Solution and Some of their Applications, Academic Press, Cambridge, MA, USA, 1999.

[47] I. Podlubny, "Fractional-order systems and $P I^{\lambda} D^{\mu}$-controllers," IEEE Transactions on Automatic Control, vol. 44, no. 1, pp. 208-214, 1999.

[48] I. Podlubny, Fractional Differential Equations, Academic Press, Cambridge, MA, USA, 1999.

[49] C.-K. Lai and K.-K. Shyu, "A novel motor drive design for incremental motion system via sliding-mode control method," IEEE Transactions on Industrial Electronics, vol. 52, no. 2, pp. 499-507, 2005.

[50] M. Fliess and C. Join, "Model-free control," International Journal of Control, vol. 86, no. 12, pp. 2228-2252, 2013.

[51] J. Liu, F.-H. Zhou, C.-C. Zhao, and Z.-R. Wang, "A PI-type sliding mode controller design for PMSG-based wind turbine," Complexity, vol. 2019, Article ID 2538206, 12 pages, 2019.

[52] S. Yu, M. Xie, H. Wu et al., "Composite proportional-integral sliding mode control with feedforward control for cell puncture mechanism with piezoelectric actuation," ISA Transactions, 2020.

[53] N. Z. Davijani, G. Jahanfarnia, and A. E. Abharian, "Nonlinear fractional sliding mode controller based on reduced order FNPK model for output power control of nuclear research reactors," IEEE Transactions on Nuclear Scienc, vol. 64, no. 1, pp. 713-723, 2017.

[54] E. Govindha Kumar and J. Arunshankar, "Control of nonlinear two-tank hybrid system using sliding mode controller with fractional-order PI-D sliding surface," Computers \& Electrical Engineering, vol. 71, pp. 953-965, 2018.

[55] R. Sadeghi, S. M. Madani, M. Ataei, M. R. Agha Kashkooli, and S. Ademi, "Super-twisting sliding mode direct power control of brushless doubly fed induction generator," IEEE Transactions on Industrial Electronics, vol. 65, no. 11, pp. 9147-9156, 2018.

[56] J. Lu, M. Savaghebi, A. M. Y. M. Ghias, X. Hou, and J. M. Guerrero, "A reduced-order generalized proportional integral observer-based resonant supertwisting sliding mode control for gridconnected power converters," IEEE Transactions on Industrial Electronics, p. 1, 2020.

[57] G. Zhang, G. Wang, B. Yuan, R. Liu, and D. Xu, "Active disturbance rejection control strategy for signal injectionbased sensorless IPMSM drives," IEEE Transactions on Transportation Electrification, vol. 4, no. 1, pp. 330-339, 2018. 